\title{
Robust ferromagnetism in wafer-scale monolayer and multilayer $\mathrm{Fe}_{3} \mathrm{GeTe}_{2}$
}

\author{
Ryan Roemer $\mathbb{D}^{1,2}$, Chong Liu (iD) ${ }^{1,2}$ and Ke Zou (iD) ${ }^{1,2 凶}$
}

Monolayer iron germanium telluride $\mathrm{Fe}_{3} \mathrm{GeTe}_{2}$, one of the typical two-dimensional ferromagnetic materials, hitherto, has only been studied by exfoliated micron-sized samples. We achieve high-quality wafer-scale growth of thin $\mathrm{Fe}_{3} \mathrm{GeTe}_{2}$ films by molecular beam epitaxy, greatly expanding the types of characterization tools employable and providing the possibility for its integration in devices like consumer electronics. Thickness-dependent transport measurements are used to characterize and probe for magnetic order. Ferromagnetic states exist in 1-10 layer thick $\mathrm{Fe}_{3} \mathrm{GeTe}_{2}$, with Curie temperatures ranging from $\sim 75 \mathrm{~K}$ in one layer samples to above $175 \mathrm{~K}$ in ten layer samples. A single ferromagnetic phase with significant magnetic anisotropy is revealed for all layer numbers. We submit the capability of synthesizing, wafer-scale $\mathrm{Fe}_{3} \mathrm{GeTe}_{2}$ as an essential step towards its fulfillment in any applications involving magnetism, such as spintronics.

npj 2D Materials and Applications (2020)4:33; https://doi.org/10.1038/s41699-020-00167-z

\section{INTRODUCTION}

The integration of magnetic materials into electronic and photonic devices has been taking place for decades ${ }^{1-3}$. One well-known example is the discovery of giant magnetoresistance and its applications ${ }^{4}$. Much research has focused on engineering new magnetic structures, especially in two-dimensions (2D). Prospects of which are plentiful in device application, not only in replacing magnets in current applications, but also in new structures, such as, in magnetic tunnel junctions and magnetic random access memories (MRAM) ${ }^{5,6}$.

The discovery of graphene ${ }^{7}$ inspired a new generation of materials known as van der Waals (vdW) materials with intrinsic 2D nature ${ }^{8}$. Samples were often accessible by exfoliating bulk crystals. After processed as a device, the electronic properties of these 2D materials could be controlled via strain, doping, gating, as well as "stacking" ${ }^{9}$ to reveal exotic states leading to an era of "vdW materials by design"10. Fundamental physical properties have been realized that were proposed long-ago ${ }^{11,12}$, in $2 \mathrm{D}$ vdW materials, including the $2 \mathrm{D}$ superconductivity found in thin films of $\mathrm{NbSe}_{2}{ }^{13}, \mathrm{FeSe}^{14}, \mathrm{WTe}_{2}{ }^{15}$, and $\mathrm{Bi}_{2} \mathrm{Sr}_{2} \mathrm{CaCu}_{2} \mathrm{O}_{8}{ }^{16}$ and so on. New opportunities in applications and a new generation of devices have become available due to the properties found in reduced dimensions.

Exfoliated 2D flakes of $\mathrm{Crl}_{3}{ }^{17}, \mathrm{Fe}_{3} \mathrm{GeTe}_{2}{ }^{18}, \mathrm{Cr}_{2} \mathrm{Ge}_{2} \mathrm{Te}_{6}{ }^{19}$, and others $^{20}$ have shown clear long-range magnetic order that are promising for applications involving properties such as aboveroom-temperature Curie temperature $\left(T_{C}\right)$ and antiferromagnetic interlayer exchange. Yet, scalability and poor stability continue to hinder the progression toward the realization of the industrialscale production of such devices ${ }^{20}$. To date, the synthesis of many 2D magnetic materials still is all limited to micron-sized flakes ${ }^{21}$, eliminating any production scale applications. Here we focus on the wafer-scale synthesis of the vdW layered ferromagnetic material $\mathrm{Fe}_{3} \mathrm{GeTe}_{2}$ (FGT), which has a $T_{\mathrm{C}}$ above room temperature $^{18}$ and is one of the few $2 \mathrm{D}$ magnetic materials that show evidence of topological states 22,23 .
Single-crystal FGT was synthesized in $2006^{24}$, revealing a relatively high $T_{\mathrm{C}} \sim 220 \mathrm{~K}$ in bulk, in comparison to the absence of magnetism in the isostructural $\mathrm{Ni}_{3} \mathrm{GeTe}_{2}$. This material has an out-of-plane magnetization vector with respect to the $\mathrm{vdW}$ planes ${ }^{25}$, showing a large anisotropy defined as the energy dependence on each magnetization vector direction. Calculations indicate its easy axis is stabilized by a large magnetocrystalline anisotropy caused by spin-orbit coupling ${ }^{26}$. Angle-resolved photoemission spectroscopy (ARPES) measurements imply the FGT bulk belongs to a class of topological nodal line semimetals ${ }^{27}$. Another ARPES experiments suggest the ferromagnetic state is accompanied by a heavy-fermion state, which has only been seen in $4 \mathrm{f}$ and beyond electron systems ${ }^{28}$.

Exfoliated FGT monolayers and multilayers ${ }^{18,29}$ preserve the characteristic ferromagnetic order of bulk. $T_{C}$ reaches $310 \mathrm{~K}$ upon ionic liquid gating in trilayer samples ${ }^{18}$, one of the highest ferromagnetic transition recorded in the $2 \mathrm{D}$ nature. Unlike $\mathrm{Crl}_{3}$, no interlayer exchange coupling has been observed in FGT films. It has been shown that FGT hosts a large Berry curvature, which is responsible for the anomalous Hall effect ${ }^{27}$. Thin-film measurements substantiate claims of topological properties in the observation of Néel-type skyrmions in FGT/WTe 2 heterostructures and large anomalous Nernst effect ${ }^{23,30}$.

To date, FGT film remains the only metallic, rare-earth free, and layered ferromagnet with out-of-plane magnetization in 2D. Because of the metallic characteristics, its fundamental magnetic properties can be studied via transport measurements, together with optical measurements such as the magneto-optic Kerr effect. Metallicity also enables easy control of charge as well as spin degrees of freedom by electrical currents, useful in device applications. For example, FGT films have been made into ultrathin devices, realizing current control of the perpendicular magnetization via spin-orbit torque, with efficiencies comparable to that of $3 \mathrm{~d}$ ferromagnetic heterostructures-the current leader of the field ${ }^{31}$. This makes FGT all the more desirable for MRAM ${ }^{32}$. Closely related application prospects are found in magnetic tunnel junction heterostructures of FGT/graphite/FGT, which display 
a
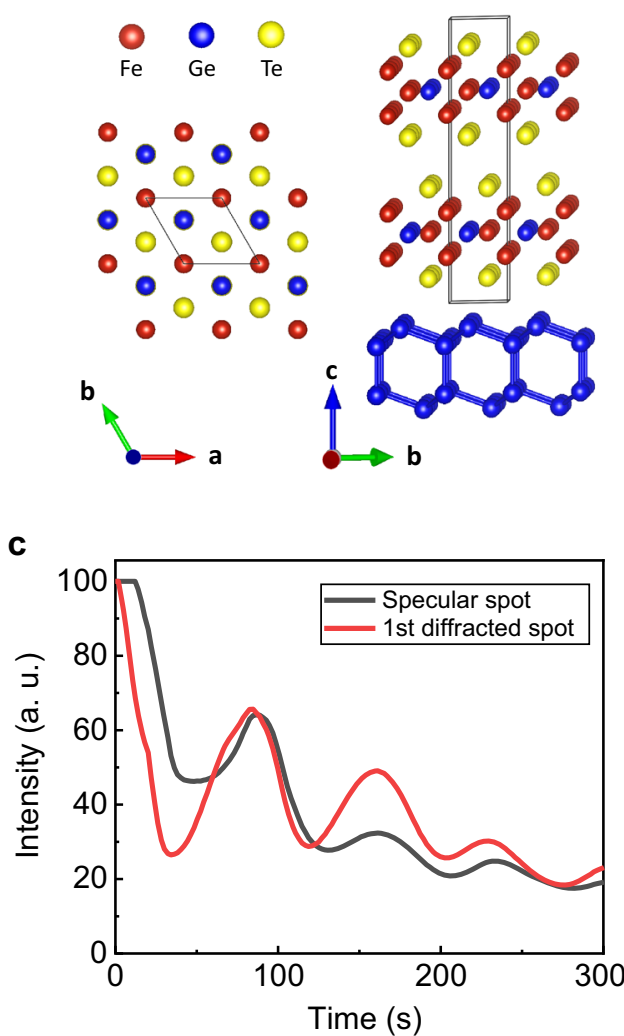

b

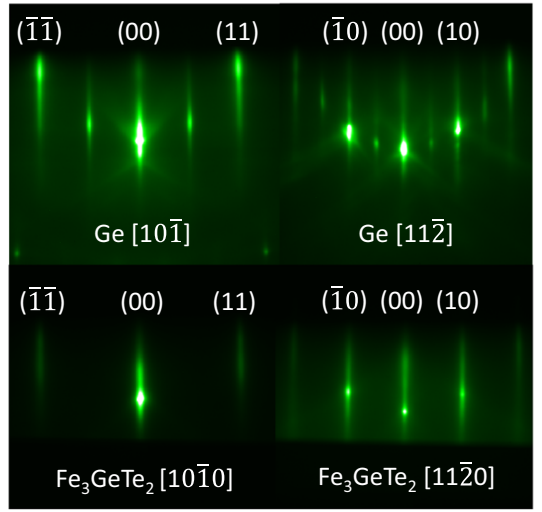

d

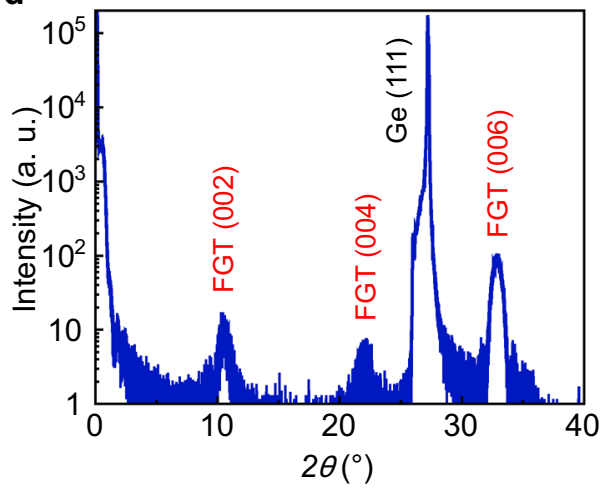

Fig. 1 Structural analysis of the high-quality $\mathrm{Fe}_{3} \mathrm{GeTe}_{2}$ thin films. a Lattice structure of $\mathrm{Fe}_{3} \mathrm{GeTe}_{2}$ (FGT), where a single unit cell (two quintuple layers $(\mathrm{QL})$ ) is outlined. The arrows indicate the local magnetic moments on the Fe atoms. b Top: in situ reflection high-energy electron diffraction (RHEED) images of a $2 \times 2$ reconstructed Ge (111) substrates along the [101] and [11 $\overline{2}$ ] direction. Bottom: 4-QL Fe ${ }_{3} \mathrm{GeTe}_{2}$ with electron beam along [1010] direction and [11 20 ] direction. c RHEED intensity oscillations during the growth of $\mathrm{Fe}_{3} \mathrm{GeTe}_{2}$, indicating atomic smoothness and its epitaxial growth mode. Single QL growth times are extracted from the period of oscillation to be $\sim 80 \mathrm{~s}$. $\mathbf{d}$ X-ray diffraction pattern of 18-QL FGT film grown on Ge (111), indicating the films are grown in the (001) direction.

promising characteristics of $160 \%$ tunneling magnetoresistance at low temperature ${ }^{33}$.

We employ molecular beam epitaxial (MBE) technique to synthesize atomically smooth and single crystalline FGT films, with thickness ranging from one quintuple layer $(\mathrm{QL}, \sim 0.8 \mathrm{~nm})$ to ten QLs on $5 \times 5 \mathrm{~mm}$ substrates. Among reported monolayer samples, ours display the highest $T_{\mathrm{c}}$ probed by anomalous Hall measurements, together with an order of magnitude larger coercivity ${ }^{18,34}$. Large switching ratios have been found in all samples at low temperatures as well as significant magnetocrystalline anisotropy energy. Our wafer-scale samples yield the opportunity for measurements and applications, which can be extremely difficult on the micron-sized flakes yielded by exfoliation techniques, such as ARPES and $x$-ray spectroscopies. One can begin integrating FGT films into commercial devices following the established techniques of $\mathrm{Si}$.

\section{RESULTS}

Structural analysis of $\mathrm{Fe}_{3} \mathrm{GeTe}_{2}$ films

The FGT unit cell is described by a hexagonal symmetry (space group $\mathrm{P}_{3} / \mathrm{mmc}$ ) composed by two weakly coupled quintuple layer $(\mathrm{QL})$ substructures, with bulk lattice parameters $a=b=$ $3.99 \AA$ and $c=16.33 \AA^{24}$, as shown in Fig. 1a. The middle three layers of each QL form a heterometallic slab of $\mathrm{Fe}^{2+} \mathrm{Ge}^{4-}$ surrounded by $\mathrm{Fe}^{3+}$ atoms. Encapsulating the slabs are tellurium layers. Theoretical calculations of monolayer magnetic moments yield $\mathrm{Fe}^{3+}$ and $\mathrm{Fe}^{2+}$ atoms contribute 1.723 and $1.005 \mu_{\mathrm{B}}$, respectively, where $\mu_{\mathrm{B}}$ is the Bohr magneton ${ }^{26}$.
We have optimized high-quality growth of epitaxial FGT(0001) ultrathin films with thicknesses ranging from $1 \mathrm{QL}$ to $10 \mathrm{QL}$ on atomically smooth $\mathrm{Ge}(111)$ substrates (See Methods). Reflection high-energy electron diffraction (RHEED) images of the $\mathrm{Ge}$ substrate and the 4-QL FGT are shown in Fig. 1b. For a hexagonal lattice, to which FGT and Ge(111) belong, there exist two distinct crystallographic directions separated by 30 degrees, the [112] and [101] directions. It is essential to note the diffraction patterns of the two crystallographic directions of the substrate and film are aligned, which confirms an epitaxial growth (Fig. 1b). Observations of intense, point-like diffraction spots indicate an atomically smooth surface of both Ge and FGT. In-plane lattice parameters are measured in situ throughout the growth process using RHEED line intensity profiles of the associated diffraction patterns. The lattice constant of the FGT film $3.99 \AA$ agrees with the known inplane lattice constant for bulk $\mathrm{Ge}(111)$, as well as the bulk FGT measurements ${ }^{24}$. A layer-by-layer growth mode is established via observation of RHEED intensity oscillations (Fig. 1c). The oscillation period allows the precise control of the number of layers deposited (Fig. 1c). Thus we confirm single-crystalline, atomically flat, and epitaxial growth of wafer-scale thin FGT films.

The Lattice structure of FGT films were further characterized by ex situ x-ray diffraction (XRD). A single phase of FGT (001) films is confirmed by the (002) family of diffraction peaks in Fig. 1d. The diffraction pattern suggests a out-of-plane lattice constant $c=$ $16.355 \AA$, in good agreement with bulk measurements of $16.33 \AA^{24}$. Fe vacancies in the $\mathrm{Fe}^{2+}$ site have been shown to decrease in-plane lattice constants and increase $c^{35}$, which are not observed in our films. 


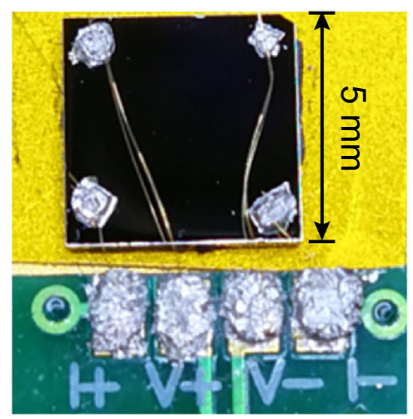

C

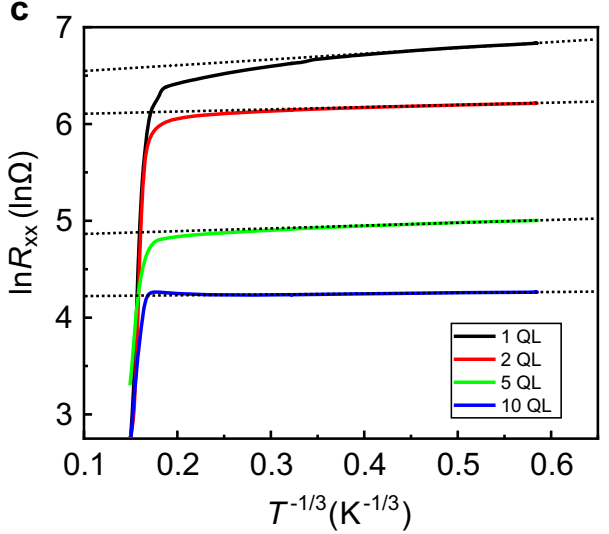

b

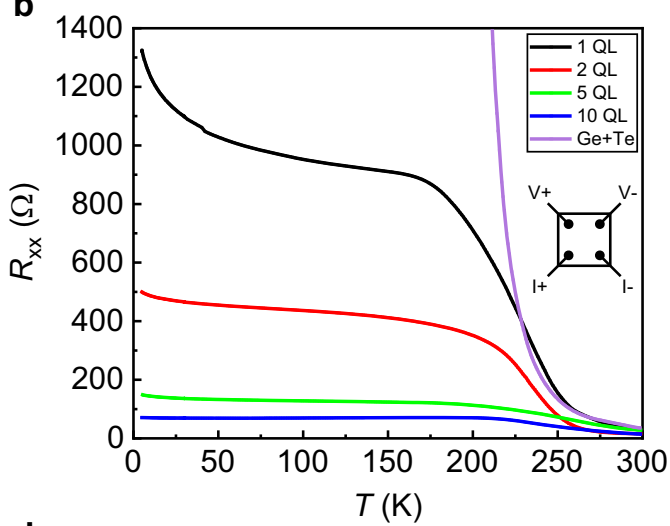

d

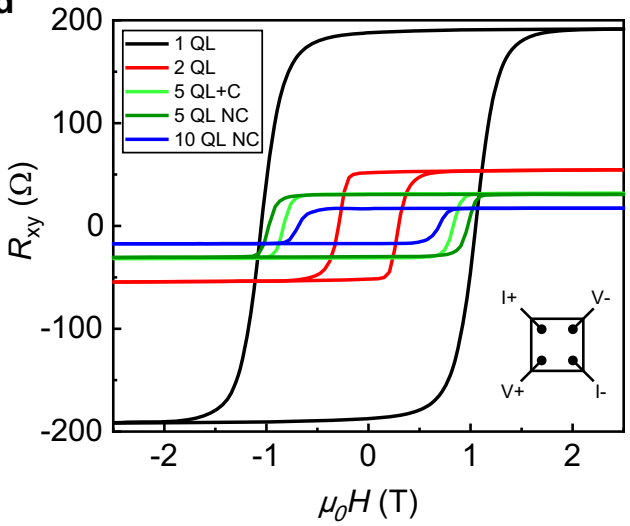

Fig. 2 Transport properties of wafer-scale FGT films. a A photo of an FGT sample measured with van der Pauw geometry. b Longitudinal resistivity $R_{\mathrm{xx}}$ vs. temperature $T$ taken of various QL numbers and capped substrate. c Fit of the $R_{\mathrm{xx}}$ with the Mott variable range hopping model, indicating the existence of localized states. d $R_{\mathrm{AH}}$ vs. applied field of select QL numbers at $5 \mathrm{~K}$ showing the anomalous Hall and hysteresis loops. The applied magnetic field $H$ is perpendicular to the sample surface and parallel to the $c$ axis of FGT. All samples are capped except the two samples marked as non-capped (NC). Insets in $\mathbf{b}$, $\mathbf{d}$ are the respective measurement geometry.

Transport results showing the conductivity and ferromagnetism in $\mathrm{Fe}_{3} \mathrm{GeTe}_{2}$ films

Electronic and magnetic properties of FGT films were characterized by transport measurements. We measure the entire wafer (Fig. 2a) with no lithography involved. Longitudinal and transverse resistances $R_{\mathrm{xx}}$ and $R_{\mathrm{xy}}$ (Fig. $2 \mathrm{~b}-\mathrm{d}$ ) were measured on both capped and uncapped samples ranging from $1 \mathrm{QL}$ to $10-\mathrm{QL}$ films. Samples are capped unless otherwise stated in the following text (see Methods regarding the capping layer). We note uncapped monolayer and bilayer films did not permit transport measurements due to insulating behavior, likely due to the oxidization of the FGT surface ${ }^{36}$. Differences in transport behavior are negligible between capped and uncapped samples when the thickness $\geq 5$ QL (Fig. 2d).

$R_{\mathrm{xx}}$ measurements show resistance values consistent with exfoliated samples ${ }^{18}$ and indicate a weak insulating behavior at low temperatures (Fig. 2b). Below $200 \mathrm{~K}, R_{\mathrm{xx}}$ fit the variable range hopping model in 2D (Fig. 2c), where $R_{x x} \propto \mathrm{e}^{T^{-1 / 3}}$. This is in agreement with previous transport studies ${ }^{37}$, which could be attributed to the emergence of localized electrons with decreasing temperature. These localized states likely originate from the Fe $3 d$ orbitals, which are also responsible for the itinerant electrons. The transition temperature agrees well with previous reports of a heavy-fermion state ("localized") in bulk ${ }^{28}$ and correlated electrons.

In control samples of Te capped Ge substrates (as shown by the purple line in Fig. 2b), we observe a stronger insulating behavior and a much higher resistance than FGT films at low temperatures.
Above $200 \mathrm{~K}$, the conduction through Ge substrates is responsible for the higher temperature transition in $R_{\mathrm{xx}}$.

To characterize intrinsic magnetic order, transverse resistance $\left(R_{\mathrm{xy}}\right)$ measurements that host the anomalous Hall ( $\left.\mathrm{AH}\right)$ effect were carried out at $5 \mathrm{~K}$, as shown in Fig. $2 \mathrm{~d}$. Here the $R_{\mathrm{AH}}$ is extracted from $R_{\mathrm{xy}}=R_{\mathrm{N}} H+R_{\mathrm{AH}}$, where $R_{\mathrm{N}}$ is the normal Hall coefficient, and $H$ is the applied field. At $5 \mathrm{~K}$, all samples display clear hysteresis indicating ferromagnetic order. 5-QL capped and uncapped samples are shown in Fig. $2 \mathrm{~d}$, revealing an almost identical remanent $\left(R_{\mathrm{R}}\right)$ and saturation resistance $\left(R_{\mathrm{S}}\right)$. This implies that, in uncapped samples above $5 \mathrm{QL}$, the top 1-2 FGT oxide layers do not significantly affect transport properties of the underneath pristine FGT layers, suggesting the electronic channels are not confined to the surface. This result is significant considering previous reports of antiferromagnetic coupling between oxidized and pristine FGT, where one would expect uncapped samples to display a two-part hysteresis. We do not observe such a superposition in hysteresis shape.

Temperature dependence of the anomalous Hall behavior Temperature-dependent $R_{\mathrm{AH}}$ measurements were also carried out on all samples from $5 \mathrm{~K}$ to $225 \mathrm{~K}$ to study the temperature evolution of the $\mathrm{AH}$ effect, as presented in Fig. 3. The observed textbook-like square shape of the hysteresis is a good indication of strong perpendicular magnetic anisotropy (PMA). It is important to note that PMA and hysteresis width are proportional ${ }^{38}$. It is also indicative of a single domain ${ }^{38}$, where all Ising like spins coherently flip at the coercive field $\left(H_{c}\right)$ along the easy axis. 

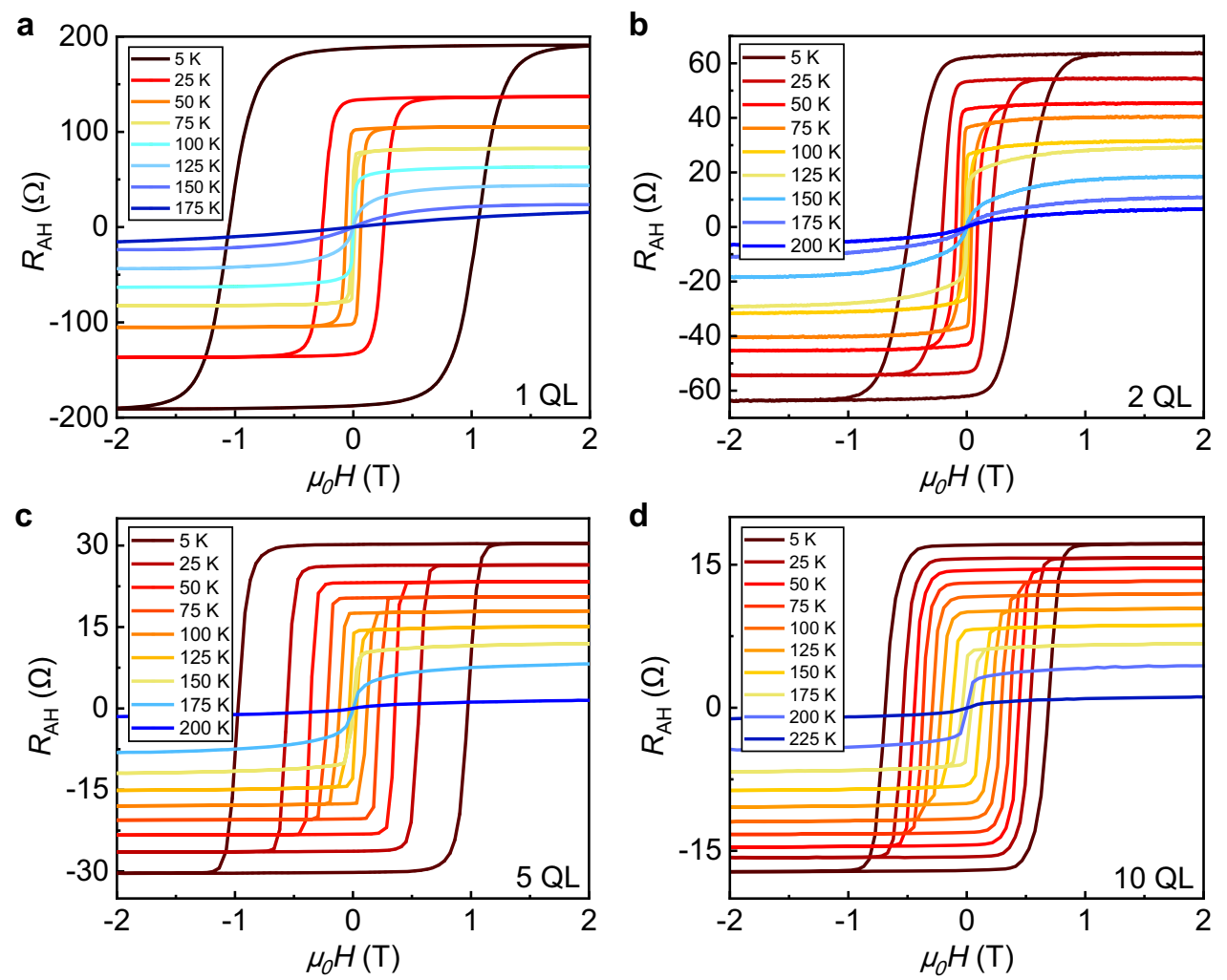

Fig. 3 Temperature-dependent ferromagnetism in FGT films. Anomalous Hall resistance of a Single-QL, b 2-QL, c 5-QL, and d 10-QL FGT films. The hysteresis loop is apparent in all samples at low temperatures and disappears as the temperature reaches the Curie temperature $T_{\mathrm{c}}$.

Analysis of the anomalous Hall results

We extract the coercive field and the remanent and saturation resistance of samples at various temperatures (Fig. 4). The coercive field $\left(H_{c}\right)$ is defined as the value of the applied magnetic field at which the loop's curve crosses through $R_{\mathrm{AH}}=0$. The remanent and saturation resistance $R_{\mathrm{R}}$ and $R_{\mathrm{S}}$ are defined as the value of $R_{\mathrm{AH}}$ at zero applied field and upon saturation, respectively. It is well established that $R_{\mathrm{R}}$ and $R_{\mathrm{S}}$ are directly proportional to the magnetic remanence $M_{R}$ (the magnetic signal at zero applied field) and magnetic saturation $M_{S}$ (the magnetic signal when all magnetic moments are maximally aligned) ${ }^{38}$. To investigate the layer and temperature-dependent evolution of the magnetic phases, we compare $R_{\mathrm{R}}, R_{\mathrm{S}}$, and $H_{\mathrm{C}}$ as a function of layer number at a fixed temperature. With increasing layer number, all samples decrease in the remanent $M_{R}$ and saturation magnetization $M_{S}$ (Fig. $4 \mathrm{a}, \mathrm{b}$ ) at a fixed temperature below the respective $T_{\mathrm{C}}$. This progression is consistent with previous reports ${ }^{18}$.

The ratio of $R_{\mathrm{R}}$ and $R_{\mathrm{S}}$ is plotted in Fig. 4c. The ratio remains nearly 1 for all QL numbers up to $100 \mathrm{~K}$, where the ratios for 5- and $10-\mathrm{QL}$ samples exceed 0.9 at room temperature. A ratio near unity is an attractive quality for storage and spintronic applications ${ }^{39}$, such as MRAM. We also note that the energy loss decreases as the layer number is increased, as indicated by the area enclosed by the hysteresis loop after a complete magnetic field sweep.

The field needed to reverse the direction of magnetization, known as the coercive field $H_{c}$, provides valuable information for assessing the stability of magnetic structures and is often used as an indication of the hardness of the magnetic domains (Fig. $4 \mathrm{~d}$ ). $H_{\mathrm{c}}$ in monolayer samples is greater than $1 \mathrm{~T}$ and roughly $0.5,0.8$, and $0.6 \mathrm{~T}$ for 2,5 , and $10 \mathrm{QL}$, respectively. Bilayer samples do not fit into a monotonic decrease of $H_{c}$ with respect to layer number. One possible explanation is related to hole doping by $\mathrm{Fe}$ vacancies, which has been shown to decrease the perpendicular anisotropy ${ }^{40}$. A more thorough layer dependent study is required to reveal the underlying cause. The spin flip transition shown by the gradual progression of $R_{\mathrm{AH}}$ around the coercive field could be due to a lack of magnetic anisotropy or the existence of multiple magnetic domains.

We use two methods to extract $T_{\mathrm{C}}$. The remanent resistance $R_{\mathrm{R}}$ serves as a direct probe of spontaneous magnetic order $M_{R}$ and allows us to extract $T_{C}$ of samples by tracking the temperature at which $R_{\mathrm{R}}$ vanishes (Fig. 4f). $T_{\mathrm{C}}$ monotonically increases with layer number. For 1, 2, 5, and 10-QL transitions take place at roughly 75, 125,150 , and $175 \mathrm{~K}$, respectively. A secondary method to extract $T_{C}$ is by Arrott plot analysis ${ }^{39}$ (Fig. 4e). Arrott plot employ the equation $R_{A H}^{2}=\mu_{0} H / R_{A H}$, from which the high field portion is linearly flitted (inset of Fig. 4e). The intercept of $R_{A H}^{2}$ is then found for multiple temperatures and again linearly fitted as a function of temperature. The resulting temperature intercept is then used as $T_{\mathrm{C}}$. This method yields an exact temperature for $T_{\mathrm{C}}$ as compared to the range generated from $R_{\mathrm{AH}}$ measurements. Using this method, we have extracted $T_{\mathrm{C}}$ to be $126 \mathrm{~K}$ in monolayer samples, $145 \mathrm{~K}$ in bilayer samples $176 \mathrm{~K}$, and $178 \mathrm{~K}$ in 5 and $10-\mathrm{QL}$ samples, respectively. The two methods show different $T_{C}$ in $<10-\mathrm{QL}$ samples but are in agreement with $R_{\mathrm{AH}}$ measurements of 10-QL samples. Upon comparison with the exfoliated layer dependant phase diagram of Deng et al., we see a significant increase in $T_{\mathrm{C}}$ for monolayer and bilayer samples (roughly $100 \mathrm{~K}$ increase), but good agreement for 5- and 10-QL samples.

\section{DISCUSSION}

We note that for all layer numbers, a strong anisotropy is maintained as indicated by the shape of the hysteresis, implying that there is limited hole doping, which has been shown to decrease the MAE and lead to small coercive fields ${ }^{40}$. This is corroborated by bulk sample measurements, which also show strong perpendicular anisotropy ${ }^{29}$. Ultrathin UHV-grown 
a

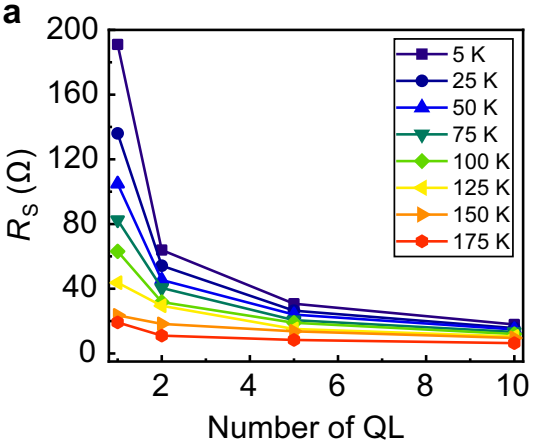

d

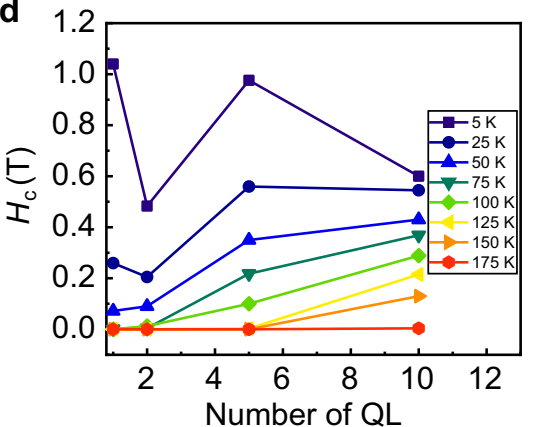

b

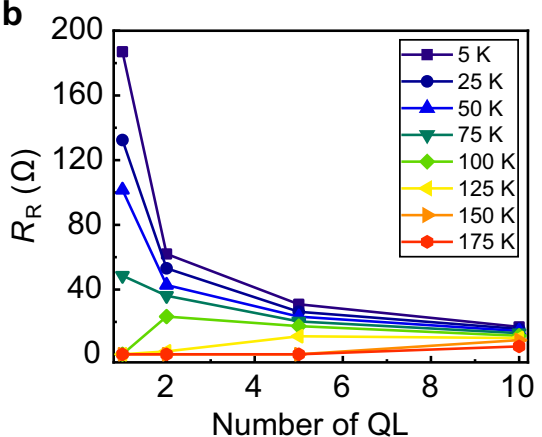

e

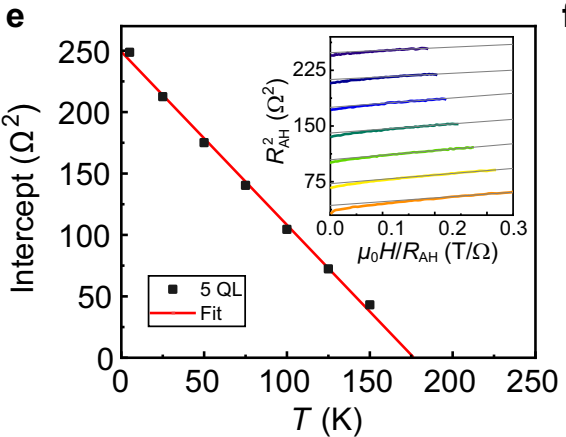

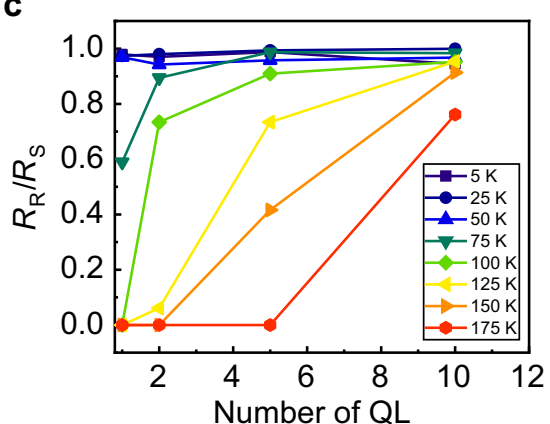

f

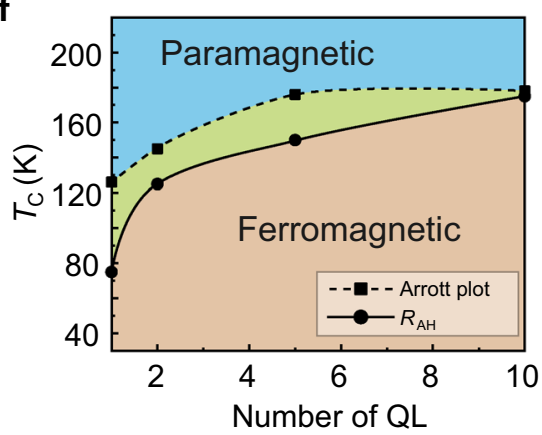

Fig. 4 Analysis of the ferromagnetisms in 1, 2, 5, and 10-QL samples. a Remanent resistance $\left(R_{\mathrm{R}}\right)$ and $\mathbf{b}$ Saturation resistance $\left(R_{\mathrm{S}}\right)$ plotted against QL number at various temperatures. Both exhibit a monotonic decrease with increasing layer numbers. c $R_{\mathrm{R}} / R_{\mathrm{S}}$ ratios remain $\sim 1$ at different temperatures, suggesting FGTs viability in applications. d Coercive field $\left(H_{c}\right)$ of various QL layer numbers. e Arrott plot analysis of a 5QL sample, indicating a $T_{C}$ of $176 \mathrm{~K}$ extracted by the $\mathrm{x}$-intercept of the fitted line. $\mathbf{f}$ Magnetic phase diagram of FGT as a function of QL number. $T_{\mathrm{C}}$ on the $R_{\mathrm{AH}}$ method line is chosen at the lowest temperature at which $R_{\mathrm{R}}$ vanishes in $\mathrm{AH}$ measurements. Arrott plot points are found using methods as in e.

monolayer samples seem to be of significantly higher $T_{\mathrm{C}}$ and more robust ferromagnetism compared to exfoliated samples, as indicated by $H_{\mathrm{C}}, R_{\mathrm{R}} / R_{\mathrm{S}}$ ratio, and the field at which samples saturate indicated by a flat $\mathrm{AH}$ signal. This is most obvious upon comparison to monolayer hysteresis reported by ref. ${ }^{18}$, for example, the coercivity of our epitaxial samples is nearly an order of magnitude larger than exfoliated samples. For monolayer, magnetic saturation was not achieved past $10 \mathrm{~T}$ while saturation was reached at only $2 \mathrm{~T}$ in exfoliated samples, yet bilayer coercivity samples agree. At low temperatures, the magnetic remanence to magnetic saturation ratio is also much larger in epitaxial samples for layer number less than $5 . T_{C}$ of monolayer and bilayer samples is higher than any previously reported using anomalous Hall measurements. Considering the decrease in coercivity caused by oxidation, indicated by differences in 5-QL capped and uncapped samples, previous monolayer data could be explained by a partially oxidized sample.

The lack of apparent interlayer coupling should be discussed with respect to recent reports of systems such as $\mathrm{Crl}_{3}$, and $\mathrm{CrBr}_{3}$. It is well known that interlayer coupling is extremely sensitive to interlayer distances, which allow for orbital overlap. The considerable distance between adjacent QL containing Fe of roughly $8 \AA$ does not lend itself to strong interlayer interaction, compared to systems such as $\mathrm{CrBr}_{3}$ where the interlayer distance is smaller. A recent study using immense pressures revealed that the antiferromagnet-ferromagnet switching is not a result of the interlayer coupling, but due to stacking order differences in $\mathrm{CrBr}_{3}{ }^{41}$. Looking forward, a recent publication shows the coupling of ferromagnetism and topological states introduces controllable currents in helical edge states in $\mathrm{Crl}_{3}$ and $\mathrm{WTe}_{2}$ heterostructures ${ }^{42}$, suggesting an interesting future in the heterostucture design of 2D magnetic materials.

In conclusion, we have synthesized high-quality ultrathin epitaxial FGT (001) films ranging from monolayer to ten layers.
The ability to grow at the wafer-scale with precise control of layer number makes production-scale device fabrication a possibility. Epitaxial thin films also allow for the growth of heterostructures and interface engineering relative to stacked samples. One particularly important aspect of the growth of thin FGT is that it opens the possibility of interfacial coupling of ferromagnetism with various materials such as superconductors and topological materials.

\section{METHODS}

MBE growth

Ge (111) substrates were chosen in our experiment, which provides a hexagonal surface with in-plane lattice constants of $a=b=4.013 \AA$, resulting in a tensile strain of $0.55 \%$ with respect to FGT. We chose Ge (111) substrate to grow monolayer films by MBE for its nonmagnetic, latticematched structure, and commercial availability. Single crystalline Ge (111) substrates were prepared ex situ by a Piranha and HF etching process. Ge (111) substrates were initially etched in a 1:3 solution of hydrogen peroxide $\left(\mathrm{H}_{2} \mathrm{O}_{2}\right)$ to sulfuric acid $\left(\mathrm{H}_{2} \mathrm{SO}_{4}\right)$ for $\sim 90 \mathrm{~s}$ to remove organic materials from the surface. Substrates were subsequently rinsed and submerged into room temperature deionized (DI) water for an inconsequential amount of time. Substrates were then submerged in a 1:5 DI water hydrofluoric acid solution for $30 \mathrm{~s}$ removing the native oxide layer on the Ge (111) surface. Substrates were again rinsed with $\mathrm{DI}$ water and loaded into a Veeco GENxplor MBE system with a base pressure of $1 \times 10^{-10}$ Torr. Substrates were annealed at $480^{\circ} \mathrm{C}$ for $\sim 7 \mathrm{~min}$ upon which a $2 \times 2$ surface reconstruction was achieved. The reconstruction was not attempted, but rather invariably emerged during the annealing process. The reconstruction served as a criterion for removing oxide or impurities from the surface but the direct influence on FGT film is not clear. Ge was then deposited to improve the substrate surface. Ge buffer layers were grown at a rate of roughly $80 \mathrm{~s}$ per layer as determined by a quartz crystal microbalance (QCM). As a result, RHEED diffraction patterns were significantly improved as indicated by the intensity increase and focusing of diffraction points as shown in Supplementary Fig. 1. 
$\mathrm{Fe}_{3} \mathrm{GeTe}_{2}$ films were synthesized using a Veeco GENxplor system. Standard effusion cells are mounted at the bottom of the chamber facing the same point at the center of the chamber where the substrate is facing downwards. Source materials of $\mathrm{Fe}(99.98 \%), \mathrm{Ge}(99.999 \%)$, and $\mathrm{Te}$ (99.9999\%) were evaporated from the effusion cells at $1320^{\circ} \mathrm{C}, 1100^{\circ} \mathrm{C}$, and $325^{\circ} \mathrm{C}$, respectively. Elements were co-deposited on the substrate at $330^{\circ} \mathrm{C}$ to grow FGT. The ratio of Fe:Ge:Te was set at 3:1: 5.6 measured by QCM prior to growth. The deposition rate was $80 \mathrm{~s}$ per QL. RHEED oscillations confirmed layer-by-layer growth (Fig. 1c and Supplementary Fig. 2). After growth of FGT, $10 \mathrm{~nm}$ of Te was deposited at room temperature as a capping layer. Without capping layer, loss of RHEED pattern of FGT confirmed amorphous surface oxidation after exposure to the air for a few minutes.

\section{X-ray diffraction}

X-ray diffraction was performed in a Rigaku Smartlab system at room temperature. A parallel beam ( $\mathrm{PB}$ ) configuration was implemented with a copper anode where both $\mathrm{Ka}_{1}$ and $\mathrm{Ka}_{2}$ emission channels were used. The voltage and current were set to $40 \mathrm{kV}$ and $44 \mathrm{~mA}$, respectively.

\section{Electrical transport}

Transport measurements were carried out in a Quantum Design physical properties measurement system (PPMS), where van der Pauw geometry was used to measure $R_{\mathrm{xx}}$ and anomalous Hall response. Contacts were made via indium cold welding and attached via a gold wire. An example of the longitudinal geometry is shown in Fig. 2.

Hall resistance was measured as a function of the magnetic field at fixed temperatures. Theoretically, this should result in a transverse resistance of the form $R_{\mathrm{xy}}=R_{\mathrm{N}} \mathrm{H}+R_{\mathrm{AH}}$, where $R_{\mathrm{N}}$ is the normal Hall coefficient, and $\mathrm{H}$ is the applied field. Magnetization is then related to the second term, where $R_{\mathrm{AH}}$ is the anomalous Hall resistance, and it's proportional to $\mathrm{M}$, the magnetization of the sample.

Arrott plot analysis was done by linearly fitting the saturated portion of $R_{\mathrm{AH}}{ }^{2}$ vs. $\left(B / R_{\mathrm{AH}}\right)$. From the linear fit, it is possible to extract a $B=0$ intercept. This analysis was performed for various temperatures around $T_{c}$. These intercepts were then plotted as a function of temperature and again linearly fitted. The intercept $=0$ value was then used to indicate $T_{C}{ }^{18}$.

\section{DATA AVAILABILITY}

All data in this published article (and its Supplementary Information files) are available from the authors.

Received: 7 May 2020; Accepted: 13 September 2020; Published online: 07 October 2020

\section{REFERENCES}

1. Ortega, N., Kumar, A., Scott, J. F. \& Katiyar, R. S. Multifunctional magnetoelectric materials for device applications. J. Phys. Condens. Matter 27, 504002 (2015).

2. Joshi, V. K. Spintronics: a contemporary review of emerging electronics devices. Eng. Sci. Technol. Int. J. 19, 1503-1513 (2016).

3. Bigot, J. Y., Vomir, M. \& Beaurepaire, E. Coherent ultrafast magnetism induced by femtosecond laser pulses. Nat. Phys. 5, 515-520 (2009).

4. Dieny, B. Magnetism Giant magnetoresistance in spin-valve multilayers. J. Magn. Magn. Mater. 136, 335-359 (1994).

5. Song, C., Cui, B., Li, F., Zhou, X. \& Pan, F. Recent progress in voltage control of magnetism: materials, mechanisms, and performance. Prog. Mater. Sci. 87, 33-82 (2017).

6. Bhatti, S. et al. Spintronics based random access memory: a review. Mater. Today 20, 530-548 (2017).

7. Novoselov, K. S. et al. Electric field effect in atomically thin carbon films. Science 306, 666-669 (2004).

8. Li, X. et al. Large-area synthesis of high-quality and uniform graphene films on copper foils. Science 324, 1312-1314 (2009).

9. Cao, Y. et al. Unconventional superconductivity in magic-angle graphene superlattices. Nature 556, 43-50 (2018).
10. Das, S., Robinson, J. A., Dubey, M., Terrones, H. \& Terrones, M. Beyond Graphene: progress in novel two-dimensional materials and van der Waals solids. Annu. Rev. Mater. Res. 45, 1-27 (2015).

11. Kosterlitz, J. M. \& Thouless, D. J. Ordering, metastability and phase transitions in two-dimensional systems. J. Phys. C. Solid State Phys. 6, 1181-1203 (1973).

12. Mermin, N. D. \& Wagner, H. Absence of ferromagnetism or antiferromagnetism in one- or two-dimensional isotropic Heisenberg models. Phys. Rev. Lett. 17, 1133-1136 (1966).

13. Xi, X. et al. Ising pairing in superconducting $\mathrm{NbSe}_{2}$ atomic layers. Nat. Phys. 12, 139-143 (2016).

14. Huang, D. \& Hoffman, J. E. Monolayer FeSe on $\mathrm{SrTiO}_{3}$. Annu. Rev. Condens. Matter Phys. 8, 311-336 (2017).

15. Sajadi, E. et al. Gate-induced superconductivity in a monolayer topological insulator. Science 362, 922-925 (2018).

16. $\mathrm{Yu}, \mathrm{Y}$. et al. High-temperature superconductivity in monolayer $\mathrm{Bi}_{2} \mathrm{Sr}_{2} \mathrm{CaCu}_{2} \mathrm{O}_{8+\delta}$. Nature 575, 156-163 (2019).

17. Huang, B. et al. Layer-dependent ferromagnetism in a van der Waals crystal down to the monolayer limit. Nature 546, 270-273 (2017).

18. Deng, Y. et al. Gate-tunable room-temperature ferromagnetism in twodimensional $\mathrm{Fe}_{3} \mathrm{GeTe}_{2}$. Nature 563, 94-99 (2018).

19. Gong, C. et al. Discovery of intrinsic ferromagnetism in two-dimensional van der Waals crystals. Nature 546, 265-269 (2017).

20. Zhang, W., Wong, P. K. J., Zhu, R. \& Wee, A. T. S. Van der Waals magnets: wonder building blocks for two-dimensional spintronics? InfoMat 1, 479-495 (2019).

21. Gong, C. \& Zhang, X. Two-dimensional magnetic crystals and emergent heterostructure devices. Science 363, eaav4450 (2019).

22. Ding, B. et al. Observation of magnetic skyrmion bubbles in a van der Waals ferromagnet $\mathrm{Fe}_{3} \mathrm{GeTe}_{2}$. Nano Lett. 20, 868-873 (2020).

23. Wu, Y. et al. Néel-type skyrmion in $\mathrm{WTe} / \mathrm{Fe}_{3} \mathrm{GeTe}_{2}$ van der Waals heterostructure. Nat. Commun. 11, 3860 (2020).

24. Deiseroth, H. J., Aleksandrov, K., Reiner, C., Kienle, L. \& Kremer, R. K. Fe $\mathrm{GeTe}_{2}$ and $\mathrm{Ni}_{3} \mathrm{GeTe}_{2}$-Two new layered transition-metal compounds: Crystal structures, HRTEM investigations, and magnetic and electrical properties. Eur. J. Inorg. Chem. 2006, 1561-1567 (2006).

25. León-Brito, N., Bauer, E. D., Ronning, F., Thompson, J. D. \& Movshovich, R. Magnetic microstructure and magnetic properties of uniaxial itinerant ferromagnet $\mathrm{Fe}_{3} \mathrm{GeTe}_{2}$. J. Appl. Phys. 120, 2-7 (2016).

26. Zhuang, H. L., Kent, P. R. C. \& Hennig, R. G. Strong anisotropy and magnetostriction in the two-dimensional Stoner ferromagnet $\mathrm{Fe}_{3} \mathrm{GeTe}_{2}$. Phys. Rev. B 93, 1-7 (2016).

27. Kim, K. et al. Large anomalous Hall current induced by topological nodal lines in a ferromagnetic van der Waals semimetal. Nat. Mater. 17, 794-799 (2018).

28. Zhang, Y. et al. Emergence of kondo lattice behavior in a van der waals itinerant ferromagnet, $\mathrm{Fe}_{3} \mathrm{GeTe}_{2}$. Sci. Adv. 4, 1-9 (2018).

29. Tan, C. et al. Hard magnetic properties in nanoflake van der Waals $\mathrm{Fe}_{3} \mathrm{GeTe}_{2}$. Nat. Commun. 9, 1-7 (2018).

30. Xu, J., Phelan, W. A. \& Chien, C. L. Large anomalous Nernst effect in a van der Waals ferromagnet $\mathrm{Fe}_{3} \mathrm{GeTe}_{2}$. Nano Lett. 19, 8250-8254 (2019).

31. Alghamdi, M. et al. Highly efficient spin-orbit torque and switching of layered ferromagnet $\mathrm{Fe}_{3} \mathrm{GeTe}_{2}$. Nano Lett. 19, 4400-4405 (2019).

32. Ralph, D. C. \& Stiles, M. D. Erratum to 'spin transfer torques'. J. Magn. Magn. Mater. 320, 1190-1216 (2008).

33. Albarakati, S. et al. Antisymmetric magnetoresistance in van der Waals $\mathrm{Fe}_{3} \mathrm{GeTe}_{2} /$ graphite $/ \mathrm{Fe}_{3} \mathrm{GeTe}_{2}$ trilayer heterostructures. Sci. Adv. 5, eaaw0409 (2019).

34. Fei, Z. et al. Two-dimensional itinerant ferromagnetism in atomically thin $\mathrm{Fe}_{3} \mathrm{GeTe}_{2}$. Nat. Mater. 17, 778-782 (2018).

35. Verchenko, V. Y., Tsirlin, A. A., Sobolev, A. V., Presniakov, I. A. \& Shevelkov, A. V. Ferromagnetic order, strong magnetocrystalline anisotropy, and magnetocaloric effect in the layered telluride $\mathrm{Fe}_{3-\delta} \mathrm{GeTe}_{2}$. Inorg. Chem. 54, 8598-8607 (2015).

36. Kim, D. et al. Antiferromagnetic coupling of van der Waals ferromagnetic $\mathrm{Fe}_{3} \mathrm{GeTe}_{2}$. Nanotechnology 30, 245701 (2019).

37. L Liu, S. et al. Wafer-scale two-dimensional ferromagnetic $\mathrm{Fe}_{3} \mathrm{GeTe}_{2}$ thin films grown by molecular beam epitaxy. npj 2D Mater. Appl. 1, 1-6 (2017).

38. Nagaosa, N., Sinova, J., Onoda, S., MacDonald, A. H. \& Ong, N. P. Anomalous Hall effect. Rev. Mod. Phys. 82, 1539-1592 (2010).

39. Li, A. P., Shen, J., Thompson, J. R. \& Weitering, H. H. Ferromagnetic percolation in $\mathrm{Mn}_{\mathrm{x}} \mathrm{Ge}_{1-\mathrm{x}}$ dilute magnetic semiconductor. Appl. Phys. Lett. 86, 1-3 (2005).

40. Park, S. Y. et al. Controlling the magnetic anisotropy of the van der Waals ferromagnet $\mathrm{Fe}_{3} \mathrm{GeTe}_{2}$ through hole doping. Nano Lett. 20, 95-100 (2019).

41. Chen, W. et al. Direct observation of van der Waals stacking-dependent interlayer magnetism. Science 366, 983-987 (2019).

42. Zhao, W. et al. Magnetic proximity and nonreciprocal current switching in a monolayer $\mathrm{WTe}_{2}$ helical edge. Nat. Mater. 19, 503-507 (2020). 


\section{ACKNOWLEDGEMENTS}

This research was supported by Max Planck-UBC-UTokyo Centre for Quantum Materials, by Canada First Research Excellence Fund, Quantum Materials and Future Technologies Program, by Natural Sciences and Engineering Research Council of Canada (NSERC), and by Canada Foundation for Innovation (CFI).

\section{AUTHOR CONTRIBUTIONS}

R.R. and K.Z. designed the project. R.R. grew the films, carried out diffraction measurements, and wrote the manuscript. R.R. and C.L. performed transport measurements and analyzed the data. All authors discussed the results and commented on the manuscript.

\section{COMPETING INTERESTS}

The authors declare no competing interests.

\section{ADDITIONAL INFORMATION}

Supplementary information is available for this paper at https://doi.org/10.1038/ s41699-020-00167-z.

Correspondence and requests for materials should be addressed to K.Z.
Reprints and permission information is available at http://www.nature.com/ reprints

Publisher's note Springer Nature remains neutral with regard to jurisdictional claims in published maps and institutional affiliations.
Open Access This article is licensed under a Creative Commons Attribution 4.0 International License, which permits use, sharing, adaptation, distribution and reproduction in any medium or format, as long as you give appropriate credit to the original author(s) and the source, provide a link to the Creative Commons license, and indicate if changes were made. The images or other third party material in this article are included in the article's Creative Commons license, unless indicated otherwise in a credit line to the material. If material is not included in the article's Creative Commons license and your intended use is not permitted by statutory regulation or exceeds the permitted use, you will need to obtain permission directly from the copyright holder. To view a copy of this license, visit http://creativecommons. org/licenses/by/4.0/.

(c) The Author(s) 2020 